\title{
Conversion of iminium salts and benzylhydrazones into aromatic azines
}

\author{
Alistair M. Stephen,* Theunis G. D. van Schalkwyk, and Neil Ravenscroft \\ Department of Chemistry, University of Cape Town, Rondebosch 7701, South Africa \\ E-mail: raveneil@science.uct.ac.za
}

Dedicated to Dr Douglas Lloyd marking his $80^{\text {th }}$ birthday

\begin{abstract}
De- $N$-alkylation of iminium salts to aromatic azines, not necessarily those directly related in structure, occurs under exceptionally mild conditions. Several mechanistic pathways may be followed, among which the facile oxidation of $\mathrm{N}$-alkylhydrazones of aromatic aldehydes appears to be a key step.
\end{abstract}

Keywords: Iminium salts, benzylhydrazones, aromatic azines, de-N-alkylation

\section{Introduction}

This communication concerns an unusual observation to the effect that $N$-dealkylation of iminium salts formed from aromatic azines is apt to occur under exceptionally mild conditions. Experiments put in hand to investigate the mechanism of the process have demonstrated some of the properties of these and related compounds, the facile oxidation of aromatic aldehyde benzylhydrazones being a key feature.

\section{Results and Discussion}

These investigations began when many years ago it was shown that iminium salts could be prepared by adding salts of secondary amines to carbonyl compounds, 1 and in an extension of this work the methochloride of salicylaldazine $\mathbf{1}$ was obtained as a pale-yellow salt of m.p. $204{ }^{\circ} \mathrm{C} .2$ On storage, however, salicylaldazine itself 2 appeared as characteristic needles showing intense fluorescence upon the surface of the microcrystalline iminium salt $\mathbf{1}$. 


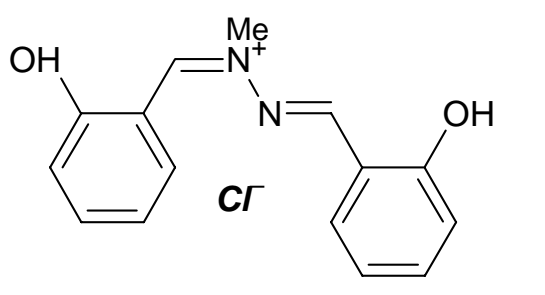

1

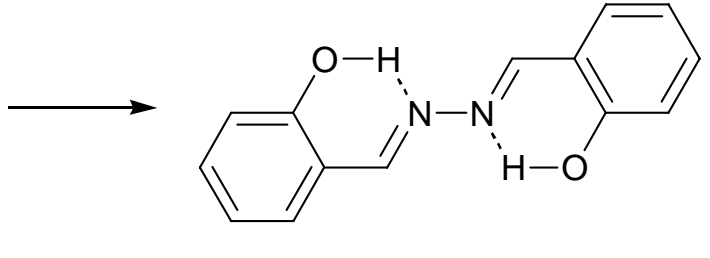

2

Figure 1 is a photomicrograph depicting needles of $\mathbf{2}$ penetrating a prismatic crystal of $\mathbf{1}$.

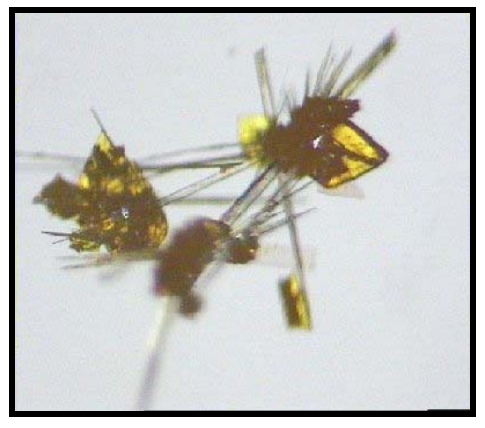

Photomicrograph depicting needles of $\mathbf{2}$ penetrating a prismatic crystal of $\mathbf{1}$

Attempted recrystallization of $\mathbf{1}$ from methanol yielded 2 . The authenticity of $\mathbf{1}$ was proved by elemental analysis and X-ray crystallography, the configuration at the iminium centre being $Z$ and that of the other moiety $E$, as for the two identical halves of salicylaldazine; in this respect some half-dozen related aromatic azines are known (CDS data file), including the prototype benzaldazine, to have the $E, E$ configuration and to be planar with respect to the aromatic rings and the azine moiety.

An exception is the o-NO2 analogue of 2 which, though E,E, is twisted in the region of the azine moiety. The EI mass spectrum of $\mathbf{1}$ indicated in addition to a strong molecular ion a preferential fission between the $\mathrm{N}$ atoms, a point of some interest when later the benzyl analogue of the metho salt 1 was examined in similar detail. TLC of 1 on Kieselgel 60 F 254 Merck using $\mathrm{CHCl} 3$ containing $\mathrm{MeOH}$ or $\mathrm{EtOH}$ showed spots (short/long wavelength UV) indicated the early formation of salicylaldehyde and its methylhydrazone $\mathbf{3}$ from $\mathbf{1}$, which was present together with a trace of the azine 2. The amount of $\mathbf{2}$ clearly increased if a sample of $\mathbf{1}$ was kept in methanol for a number of days, and, working on a larger scale, pale-yellow needles of 2, m.p. $216^{\circ} \mathrm{C}$, were obtained. As expected, the $1 \mathrm{H}$ and $13 \mathrm{C}$ NMR spectra of 1 in DMSO-d6, initially and when the solutions were kept for 9 days, were not uncomplicated and showed changes with time: salicylaldehyde and some 2 were apparent from the beginning and a number of signals due to other compounds present were observed (cf. the postulated reaction pathways given below in 
Scheme 2). Direct nucleophilic displacement of a methyl group from 1 is not considered likely in spite of the stability of $\mathbf{2}$ as a leaving group. Direct displacement of methyl from $\mathbf{1}$ would, initially at least, result in retention of a $Z$ configuration. In order
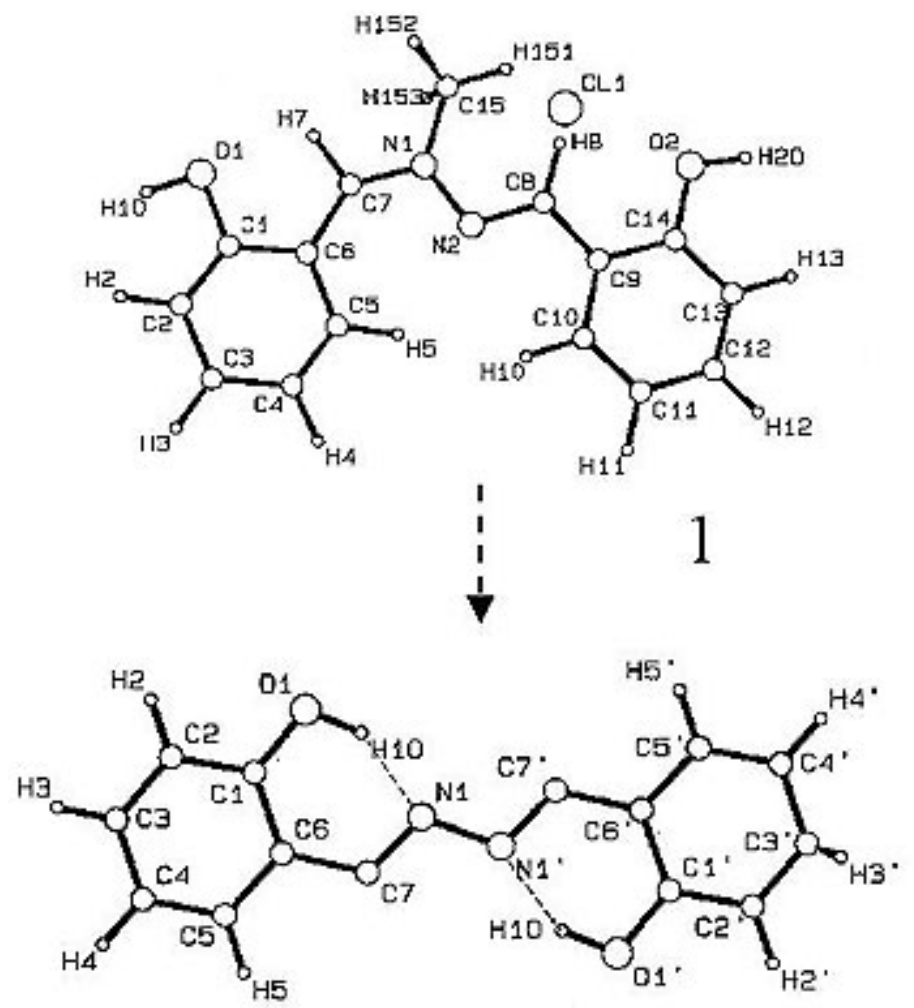

2

$\mathrm{X}$-ray structures 1 and 2<smiles>CCN(C)N=Cc1ccccc1O</smiles>

to explore further the mode(s) of detachment of the methyl carbon atom from nitrogen, experiments were conducted using $N$-benzyl rather than $N$-methyl-containing iminium salts. 


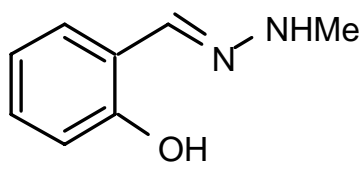

3

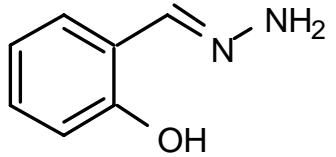

4

It should be noted first that the hydrochloride of 3, having m.p. $161{ }^{\circ} \mathrm{C}$, was converted to $\mathbf{1}$ on addition of salicylaldehyde, in 1,4-dioxane for example; and that furthermore this hydrochloride kept in DMSO showed a palpable increase with time of the amount of azine 2 present, in addition to salicyldehyde, which appeared (TLC) very soon after dissolving the salt in methanol. Crystals of $\mathbf{3}$ became coated in time with thick encrustations of the highly-fluorescent 2. The choice of solvent used to demonstrate these various transformations was largely actuated by practical considerations in setting up the TLC monitoring experiments.

Additional experiments that have a bearing on the process of azine formation were (i) the observation (TLC, and confirmed by chromatography on a larger scale) that salicylaldehyde hydrazone 4 decomposes in solution yielding both the aldehyde and, by disproportionation, azine 2; (ii) the preparation of analogues of 1 using methylhydrazine hydrochloride and p-hydroxy-, pmethoxy- or p-dimethylaminobenzaldehyde, all of which reverted to azines in the course of time after dissolution. The latter observation rules out the necessity for the o-hydroxyl group in the salicyl residue to be present, for demethylation to occur. (iii) The isolated yield of $\mathbf{2}$ from $\mathbf{1}$ kept for three weeks in DMSO solution in the open air was 20\%; in fact the bulk of the residue after solvent removal was soluble in $\mathrm{CHCl}_{3}$, and therefore was not unchanged $\mathbf{1}$.

Thereafter a series of aromatic aldehyde derivatives using benzylhydrazine dihydrochloride was prepared in methanol, with varying degrees of success. The benzyl analogue 5 of the methochloride 1, in DMSO, was then aerated typically by bubbling an air stream through the solution or passing air over the surface: the effluent gas contained benzaldehyde, which caused cloudiness to develop rapidly in an acidified 2,4-dinitrophenylhydrazine trap. During the course of three days a copious precipitate had formed, and was collected and identified as benzaldehyde DNP (m.p. and mixed m.p., TLC and HPLC). The major product remaining in the DMSO was azine 2. Iminium salts related to 5 , prepared from the para-substituted benzaldehydes containing $\mathrm{OH}, \mathrm{OMe}$, and $\mathrm{NMe}_{2}$ groups, all yielded benzaldehyde during various periods of treatment, some volatilized and some retained in the solvent. The residues were complex mixtures, as explained in the next paragraph, but the expected aromatic aldazine was observed in all cases.

It was concluded that a sequence of steps (involving tautomerization, hydrolysis and oxidation, in an acid medium) would account for the release of the benzyl substituent from its attachment to nitrogen and formation of the azine from 5 and its analogues. Confirmation of the feasibility of the oxidation step was obtained by keeping the hydrochloride of benzaldehyde 
benzylhydrazone in methanol for two weeks, the colourless solution becoming yellow rapidly due to benzaldazine formation. The identity of the azine was indicated by TLC, HPLC and the UV spectrum, and by X-ray crystallographic examination of the isolated product. Furthermore, the hydrochloride of $\mathrm{p}$-chlorobenzaldehyde benzylhydrazone after eight days in DMSO yielded crystalline p-chlorobenzaldazine 6 (48\%), and analyses showed that an estimated $65 \%$ transformation of benzyl groups to benzylidene had occurred (see Scheme 1), thus accounting for the mixtures of products in the residues after air/solvent treatment of $\mathbf{5}$ and its counterparts; azine formation resulted also from oxidation of the benzylhydrazones of p-nitrobenzaldehyde and p-tolualdehyde (hydrochlorides m.p. $172{ }^{\circ} \mathrm{C}$ and $126{ }^{\circ} \mathrm{C}$ ) treated similarly. As a practical point, it should be noted that HPLC (standard C-18 column, 85\% methanol; monitoring at 360 $\mathrm{nm}$ ) was a satisfactory means of separating and analysing quantitatively such DNP mixtures as were formed from volatile, substituted benzaldehydes accompanying benzaldehyde in the effluent gas streams; TLC on silica using chloroform /cyclohexane mixtures (typically 2:1, made basic with $\mathrm{Me}_{4} \mathrm{NOH}$ ) was used for screening DNPs and azine mixtures.

The scheme put forward for transformations of the various benzyl derivatives (Scheme 2; route A preferred because supported by collateral experiments), in any of several variants and noting the reversibility of most of the steps in an acid medium, may well apply to the initial question as to the mechanism(s) of de- $N$-methylation discussed above. However, there are differences which are apparent. For example, tautomeric shift to form a methylene isomer of $\mathbf{1}$ would be disfavoured on account of the lack of a conjugative effect such as the benzylidene group (putative route B) enjoys. On the other hand, salicylaldehyde methylhydrazone does form the azine 2. From a practical standpoint, the production from $\mathbf{1}$ of formaldehyde, isolated as it was in trace amounts in the form of its DNP, was barely measureable, though the reactivity of $\mathrm{CH}_{2} \mathrm{O}$ is such that condensation with the aromatic aldehyde present and its removal thereby from the system would be expected to have occurred rapidly. 


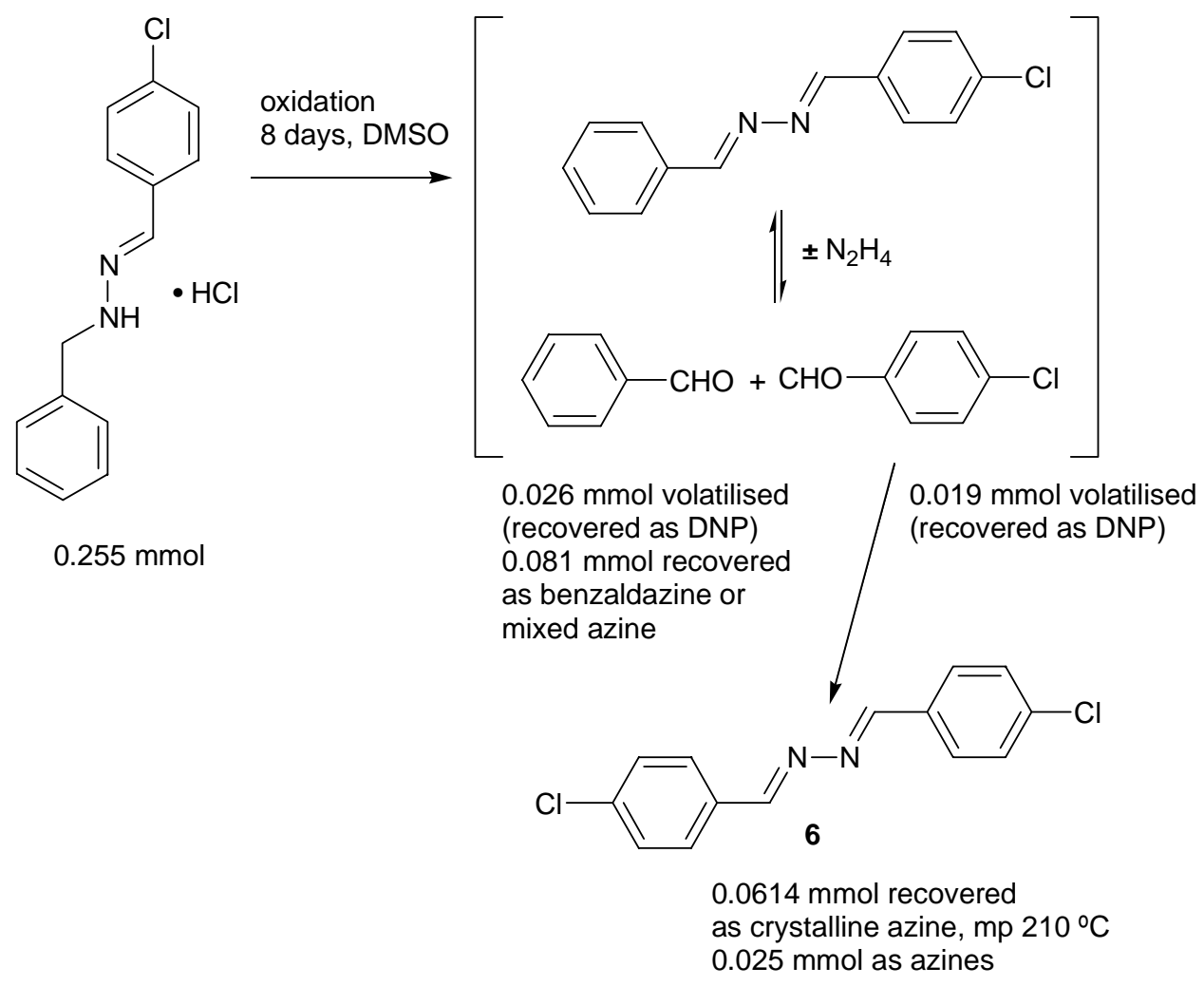

Scheme 1. Products of benzylhydrazone oxidation. 


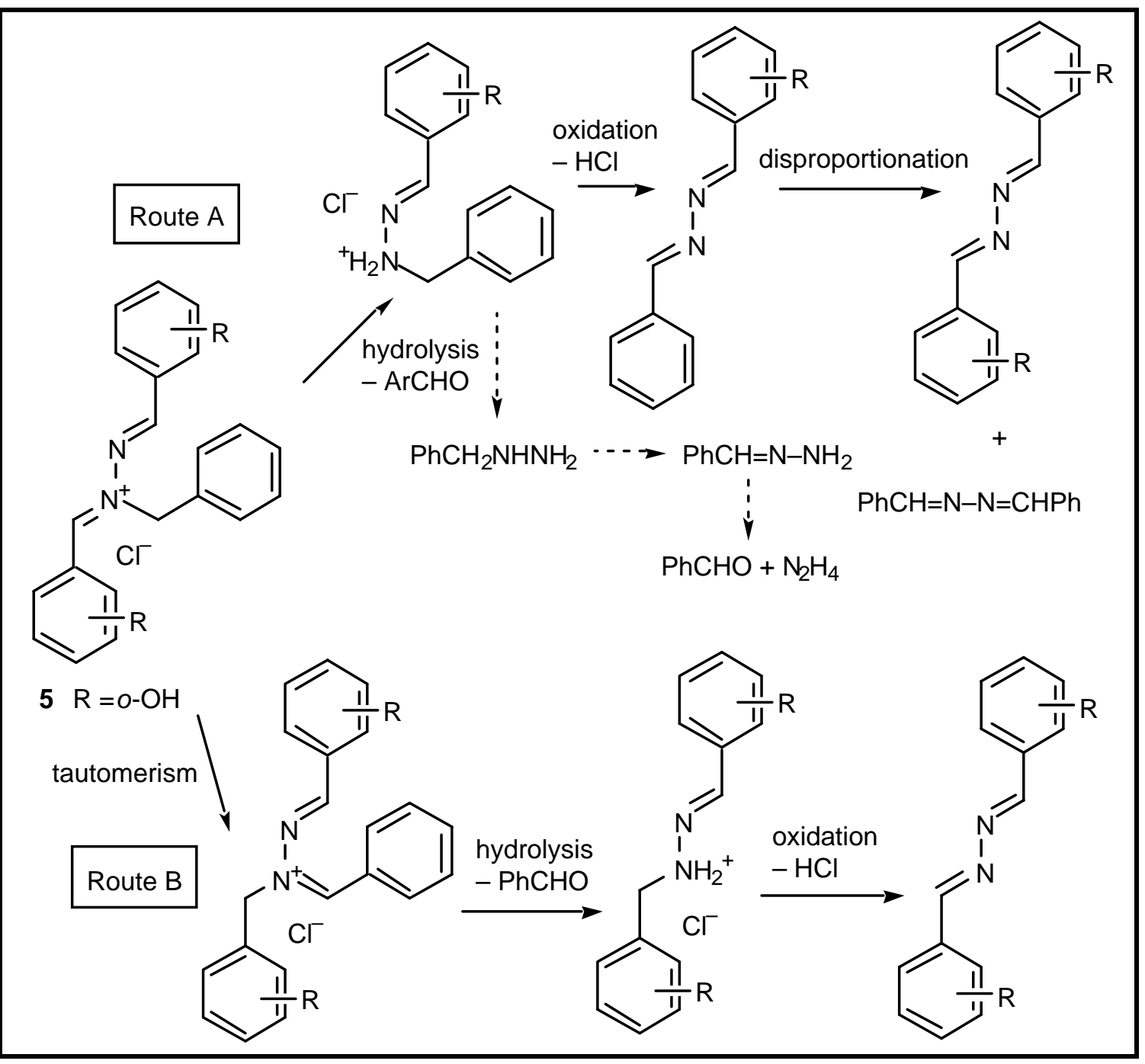

Scheme 2. Suggested pathways for $N$-debenzylation.

(NB the three dimensional structure of $\mathbf{5}$ is not yet known)

Furthermore the fragmentations of $\mathbf{1}$ and of the p-equivalent of $\mathbf{5}$ in mass spectrometry are very different, in the latter instance the pronounced stabilization of the benzyl (tropylium) cation being the cause of M-91 constituting the ion of highest $\mathrm{m} / \mathrm{z}$. It is thus evident that several mechanisms may be involved in the dealkylation processes studied.

Our most recent communication relating to this paper was "Transformations of iminium salts and benzylhydrazones into azines", by A. M. Stephen, L. R. Nassimbeni, M. R. Caira and T. G. D. van Schalkwyk, presented at the 5th Frank Warren National Organic Chemistry Conference, Aventura Aldam, Free State, South Africa, 4-7 April, 1995. An extended report "Azines of aromatic aldehydes from the methochlorides and the corresponding benzyl analogues - a novel 
$N$-dealkylation process", by the authors, is lodged in the library of the Chemistry Department. This includes inter alia a detailed description of the air-bubbling experiment using the benzylhydrazone of $p$-chlorobenzaldehyde, which led to $p$-chlorobenzaldazine and other products, and includes as well crystallographic and NMR information.

\section{Experimental Section}

General Procedures. TLC of the iminium salts and their degradation products was carried out on Kieselgel 60 F 254 Merck using $\mathrm{CHCl}_{3}$ containing 1-2\% $\mathrm{MeOH}$ or $\mathrm{EtOH}$, and viewing the developed plates under long wavelength UV (especially effective for revealing 2), or short wavelength $\mathrm{UV}$ (for aromatic moieties). $\mathrm{FeCl}_{3}$ in aqueous $\mathrm{HCl}$ was used to reveal phenolic groupings. For DNPs and aromatic azines TLC on silica was performed using $\mathrm{CHCl}_{3} /$ cyclohexane mixtures (typically 2:1, with added $\mathrm{Me} 4 \mathrm{NOH}$ ). Semi-quantitative analysis of DNPs was carried out on a standard C-18 column with $85 \% \mathrm{MeOH}$, monitoring at $360 \mathrm{~nm}$ : some retention times $T$ were, for the DNP of benzaldehyde 7.4, of salicylaldehyde 6.05 , of $p$ hydroxybenzaldehyde 4.76, of $p$-nitrobenzaldehyde 5.73 , of anisaldehyde 8.24 , of $p$ tolualdehyde 10.15 and of $p$-chlorobenzaldehyde 9.05. $T$ for formaldehyde DNP was 4.25 and for DNP itself 3.47.

NMR spectra were obtained for $1 \mathrm{H}$ at $400 \mathrm{MHz}$ using a Varian Unity 400 instrument, and for ${ }_{13} \mathrm{C}$ and ${ }_{1} \mathrm{H}-13 \mathrm{C}$ correlation experiments using a Varian Mercury 300 instrument. All spectra were run on solutions in DMSO-d6 with reference to residual DMSO-d6 signals $(1 \mathrm{H}$ at $2.50 \mathrm{ppm}$ and ${ }_{13} \mathrm{C}$ at $\left.39.52 \mathrm{ppm}\right)$. The most significant regions for ${ }_{1} \mathrm{H}$ were at $\delta 9$ (methine protons) and $\delta 3(N-$ $\mathrm{Me}$ ), and for ${ }_{13} \mathrm{C} \delta 160$ (methine $\mathrm{C}$ ) and $\delta 40(\mathrm{~N}-\mathrm{Me})$. Mass spectra were recorded on a Micromass $16 \mathrm{~F}$ spectrometer using EI at $70 \mathrm{eV}$. X-ray crystal structures were obtained on an Enraf-Nonius CAD4 diffractometer using Mo Ka radiation $(0.7107 \AA)$. The CSD data files consulted were to October 2000.

Preparation of iminium salts and hydrazones. These were prepared in $\mathrm{MeOH}$ or $\mathrm{EtOH}$ solution by mixing aldehyde with the $\mathrm{N}$-substituted hydrazine as its hydrochloride, Et $2 \mathrm{O}$ being added when necessary to achieve precipitation of the derivatives. Prolonged chilling was sometimes required. The identities of the products, as indicated in the Results section, were supported by TLC, HPLC and combinations of elemental analysis, NMR and mass spectroscopy, and in some cases X-ray crystallographic examination. 


\section{Acknowledgements}

Some of the experimental work was carried out in the laboratories of the Fishing Industry Research Institute, Rosebank, by courtesy of the Director, Professor J. P. H. Wessels, and staff. We thank the J. Murray Hofmeyr Charitable Trust for providing financial assistance.

\section{References}

1. Lamchen, M.; Pugh, W.; Stephen, A.M. J. Chem. Soc. 1954, 4418.

2. Lamchen, M.; Stephen, A. M. J. Chem. Soc. 1955, 2044. 